\title{
Finanzierung der Adipositaschirurgie: Antrag, Widerspruch und Klage
}

\author{
Tim C. Werner \\ Kanzlei Werner Rechtsanwälte, Frankfurt/M., Deutschland
}

\section{Schlüsselwörter \\ Adipositaschirurgie $\cdot$ Kostenübernahme . Gesetzliche Krankenversicherung · GKV . Rechtsschutz $\cdot$ Klage}

\section{Zusammenfassung}

Die deutsche Bevölkerung ist mit adipositaschirurgischen Verfahren unterversorgt. Dieser beklagenswerte Umstand hat seinen Grund in der restriktiven, nicht selten rechtswidrigen Genehmigungspraxis der Gesetzlichen Krankenkassen. Die Zahl der Patienten, die sich dagegen wehren, steigt stark an. Ablehnende Entscheidungen können mit einem Widerspruch und einer Klage angefochten werden. Die Rechtslage ist unklar, die Rechtsanwender verunsichert. Verschiedene Gerichte behandeln vergleichbare Sachverhalte völlig unterschiedlich. Dennoch sind die Erfolgsaussichten gut: Die deutschen Sozialgerichte entscheiden in letzter Zeit zunehmend patientenfreundlich. Die Chancen, in einem Widerspruchs- oder Klageverfahren zu obsiegen, steigen rapide an, sobald ein Body Mass Index (BMI) von $60 \mathrm{~kg} / \mathrm{m}^{2}$ erreicht ist.

\section{Die Ausgangslage}

In Deutschland sind bereits $50 \%$ der Bevölkerung übergewichtig (BMI $>25 \mathrm{~kg} / \mathrm{m}^{2}$ ); $20 \%$ sind adipös (BMI $>30 \mathrm{~kg} / \mathrm{m}^{2}$ ) [1]. Die adipösen Patienten leiden nicht selten unter gravierenden Begleit- und Folgeerkrankungen (unter anderem arterielle Hypertonie, Diabetes mellitus Typ 2, Schlafapnoe, massive Überlastung des gesamten Bewegungsapparats, reaktive Depression) und sind in Bezug auf die kardiovas-

\section{Keywords}

Obesity surgery - Meeting the costs .

German statutory health insurance . GKV .

Legal protection - Lawsuit

\section{Summary}

Funding of Obesity Surgery: Application, Appeal and Lawsuit

Due to the fact that the statutory health insurance (GKV) follows a restrictive authorisation practice the German population is undersupplied with bariatric operations. The number of patients who refuse to accept this practice increases considerably. Negative decisions can be contested by appeal and lawsuit. The legal situation is yet unclear, those applying the law, such as lawyers, judges and the health insurance authorities, are confused and disorientated. In absolutely comparable cases different courts come to different decisions. Nevertheless the topic is worth the fight: The German social courts are passing more and more patient-friendly judgements. Cases are easily won by plaintiffs if the body mass index (BMI) is higher than $60 \mathrm{~kg} / \mathrm{m}^{2}$.

kuläre Problemlage Hochrisikopatienten. Morbidität und Mortalität sind erhöht. Familiäre und soziale Bindungen lösen sich auf.

Obwohl Effizienz und Nutzen der Adipositaschirurgie mittlerweile sehr gut nachgewiesen sind [2] und obwohl sich die Zahl der Eingriffe in den letzten Jahren weltweit verdoppelt hat [3], kommen adipositaschirurgische Verfahren - trotz entsprechender medizinischer Indikation - zu selten zur Anwendung. Die Bevölkerung ist unterversorgt. Grund hierfür

\section{KARGER \\ Fax +497614520714 \\ Information@Karger.de}

www.karger.com (c) 2011 S. Karger GmbH, Freiburg

$1662-4025 / 11 / 0047-0050 \$ 38.00 / 0$

Accessible online at:

www.karger.com/ofa 
ist in erster Linie die restriktive Genehmigungspraxis der gesetzlichen Krankenkassen (GKV). Der nachfolgende Beitrag stellt die Rechtslage dar und berichtet aus der Rechtspraxis.

\section{Die Rechtsgrundlagen}

\section{§ 27 Absatz 1 SGB V (Krankenhausbehandlung)} "Versicherte haben Anspruch auf Krankenbehandlung, wenn sie notwendig ist, um eine Krankheit zu erkennen, zu heilen, ihre Verschlimmerung zu verhüten oder Krankheitsbeschwerden zu lindern.»

\section{§12 Absatz 1 SGB V (Wirtschaftlichkeitsgebot)}

«Die Leistungen müssen ausreichend, zweckmäßig und wirtschaftlich sein; sie dürfen das Maß des Notwendigen nicht überschreiten. Leistungen, die nicht notwendig oder unwirtschaftlich sind, können Versicherte nicht beanspruchen, dürfen die Leistungserbringer nicht bewirken und die Krankenkassen nicht bewilligen.»

\section{$\S 2$ Absatz 2 SGB I (Sozialstaatsprinzip)} «Die nachfolgenden sozialen Rechte sind bei der Auslegung der Vorschriften dieses Gesetzbuches und bei der Ausübung von Ermessen zu beachten; dabei ist sicherzustellen, dass die sozialen Rechte möglichst weitgehend verwirklicht werden.»

Die letztgenannte Vorschrift ist von wesentlicher Bedeutung, da sie das verfassungsrechtlich verankerte Sozialstaatsprinzip konkretisiert. Für den Bereich der GKV (SGB V) beschreibt sie einen weiten Ermessensspielraum für Krankenkassen und Gerichte und mahnt eine wohlwollende Prüfung an.

Im Unterschied zur ambulanten Versorgung ( $\$ 135$ SGB V) unterliegt die stationäre Versorgung von Patienten mit neuartigen Verfahren keinem Erlaubnisvorbehalt ( $\$ 137 \mathrm{c}$ SGB V). Auch neuartige Verfahren bedürfen keiner Zulassung und können zu Lasten der GKV angewendet werden, solange sie der gemeinsame Bundesausschuss nicht ausgeschlossen hat [4].

\section{Die Rechtsprechung}

Das Bundessozialgericht (BSG) in Kassel hat am 19. Februar 2003 sämtliche bis dato anhängigen, eine Kostenübernahme für eine Magenoperation zur Behandlung der Adipositas ablehnenden Urteile der unteren Instanzen aufgehoben und somit eine Erstattungspflicht der Krankenkassen - jedenfalls im Grundsatz - bejaht [5].

Das Gericht hat sich dabei insbesondere den Einwand der beklagten Krankenkassen verworfen, die Kosten für einen Eingriff an einem funktionell intakten Organ könnten nicht übernommen werden. Angemahnt wurde ferner die Beach- tung des Umstandes, dass chirurgische Therapien den Krankenkassen zu ganz erheblichen Einsparungen verhelfen.

Das Gericht hat insbesondere die folgenden Ausführungen gemacht:

"Eine solche mittelbare Therapie wird jedoch vom Leistungsanspruch grundsätzlich mit umfasst, wenn sie ansonsten die in $\S 2$ Absatz 1 Satz 3 und $\S 12$ Absatz 1 SGB V aufgestellten Anforderungen erfüllt, also ausreichend, zweckmäßig und wirtschaftlich ist sowie dem allgemein anerkannten Stand der wissenschaftlichen Erkenntnisse entspricht. Für chirurgische Eingriffe hat der Senat diesen Grundsatz allerdings eingeschränkt: Wird durch eine solche Operation in ein funktionell intaktes Organ eingegriffen und dieses regelwidrig verändert, wie das bei der Applikation eines Magenbandes geschieht, bedarf die mittelbare Behandlung einer speziellen Rechtfertigung, wobei die Art und Schwere der Erkrankung, die Dringlichkeit der Intervention, die Risiken und der zu erwartende Nutzen der Therapie sowie etwaige Folgekosten für die Krankenversicherung gegeneinander abzuwägen sind (...).»

"Da das Behandlungsziel einer Gewichtsreduktion auf verschiedenen Wegen erreicht werden kann, ist zunächst zu prüfen, ob eine vollstationäre chirurgische Behandlung unter Berücksichtigung der Behandlungsalternativen (diätetische Therapie, Bewegungstherapie, medikamentöse Therapie, Psychotherapie) notwendig und wirtschaftlich ist ( $12 \mathrm{Ab}$ satz 1, § 39 Absatz 1 Satz 2 SGB V). Sodann muss untersucht werden, ob nach dem aktuellen Stand der wissenschaftlichen Diskussion aus medizinischer Sicht die Voraussetzungen für eine chirurgische Intervention gegeben sind. Nach den vorliegenden Leitlinien der Fachgesellschaften (...) und den einschlägigen Literaturbeiträgen (...) kommt die Implantation eines Magenbandes nur als Ultima Ratio und nur bei Patienten in Betracht, die eine Reihe von Bedingungen für eine erfolgreiche Behandlung erfüllen: - BMI > = 40 oder > = 35 mit erheblichen Begleiterkrankungen

- Erschöpfung konservativer Behandlungsmöglichkeiten

- tolerables Operationsrisiko

- ausreichende Motivation

- keine manifeste psychiatrische Erkrankung

- Möglichkeit einer lebenslangen medizinischen Nachbetreuung".

Die Entscheidungen lassen den Krankenkassen und Gerichten einen weiten - zu weiten - Auslegungsspielraum. Die Folge ist Rechtsunsicherheit und ein Flickenteppich von Entscheidungen.

Problematisch gestaltet sich weiterhin der Umstand, dass einige Richter die vom BSG geforderte Abwägung gerade 
nicht vornehmen und fälschlicherweise davon ausgehen, dass tatsächliche (und nicht medizinische) Fragen für das streitige Verfahren entscheidungserheblich sind.

In jüngster Zeit scheinen die Gerichte aber in Richtung einer vorsichtig patientenfreundlichen Rechtsprechung umzuschwenken, zuletzt:

- LSG Niedersachsen-Bremen, Urteil vom 18. August 2010, Az. L 4 KR 169/08

- SG Lüneburg, Urteil vom 24. Februar 2011, Az. S 16 KR 202/09 (6)

- SG Frankfurt am Main, Urteil vom 30. November 2010, Az. S 25 KR 737/07

- SG Frankfurt am Main, Urteil vom 12. Oktober 2010, Az. S 25 KR 348/07

- LG Wiesbaden, Urteil vom 5. Mai 2010, Az. 5 O 263/08 (noch nicht rechtskräftig)

- SG Frankfurt am Main, Urteil vom 26. April 2010, Az. S 18 KR 634/06

- SG Münster, Urteil vom 28. Januar 2010, Az. S 11 KR 283/06

- SG Schwerin, Urteil vom 27. Januar 2010, Az. S 8 KR $57 / 08$

- SG Darmstadt, Urteil vom 3. Dezember 2009, Az. S 18 KR 418/08.

\section{Antrag, Widerspruch und Klage}

\section{Das Antragsverfahren}

Der Erstantrag bei der Krankenkasse erfolgt in der Regel durch den Patienten selbst, gegebenenfalls in Zusammenarbeit mit dem Haus- bzw. Facharzt oder dem Krankenhaus, bei welchem ein chirurgischer Eingriff geplant ist.

Die Einbeziehung eines Rechtsanwaltes als Ghostwriter ist sinnvoll, da sich falsche, taktisch unkluge oder missverständliche Aussagen stets zu Lasten des Antragstellers auswirken.

\section{Das Widerspruchsverfahren}

Gegen die Ablehnung des Antrages auf Kostenübernahme ist der Widerspruch möglich. Dieser wird direkt bei der Krankenkasse erhoben. Eine Pflicht zur Begründung des Widerspruchs existiert nicht. Über ihn entscheidet der sogenannte Widerspruchsausschuss. Es empfiehlt sich, an der abschließenden Sitzung dieses Ausschusses teilzunehmen, um den Sachverhalt persönlich vorzutragen.

Bereits in der Ablehnung muss der Versicherte auf die Möglichkeit eines Widerspruchs hingewiesen werden. Fehlt die sogenannte Rechtsbehelfsbelehrung, so verlängert sich die Frist zur Erhebung des Widerspruches von 1 Monat auf 1 Jahr (§ 66 Absatz 2 Sozialgerichtsgesetz, SGG).

Das Widerspruchsverfahren, für dessen Bearbeitung der Krankenkasse längstens 3 Monate Zeit bleiben ( 88 Absatz 2 SGG) endet entweder mit der sogenannten Abhilfeentscheidung (Kostenübernahmeerklärung) oder mit der Zurückwei- sung des Widerspruches durch den sogenannten Widerspruchsbescheid.

Spätestens im Widerspruchsverfahren ist anwaltlicher Beistand erforderlich.

Die Vorteile des Widerspruchsverfahrens sind das schnelle Verfahren, die unmittelbare Korrespondenz direkt zwischen den Parteien, die Kostenfreiheit und die guten Erfolgsaussichten.

In der Regel wird im Widerspruchsverfahren erstmals oder wiederholt ein Gutachten des Medizinischen Dienstes (MDK) eingeholt. In diesem Zusammenhang ist Folgendes $\mathrm{zu}$ beachten:

- Der Widerspruchsführer sollte persönlich angesehen werden.

- Der Widerspruchsführer sollte sich einem Coaching durch einen Arzt, eine Selbsthilfegruppe oder einen Rechtsanwalt unterziehen: Wichtig sind vor allem die sogenannte Compliance (aufgeklärter, informierter und engagierter Patient, auch und gerade in Bezug auf die Nachsorge), eine authentische Darstellung des Leidensdrucks sowie schließlich eine realistische Einschätzung des Zielgewichts.

\section{Das Verfahren vor dem Sozialgericht}

Hat der Widerspruch keinen Erfolg, so kann Klage zum Sozialgericht erhoben werden, zuständig ist immer das Gericht am Wohnort des Klägers ( $\$ 57$ SGG). Die Klage muss binnen eines Monats nach Zugang des Widerspruchsbescheids erhoben werden. War diesem Bescheid keine oder eine fehlerhafte Rechtsbehelfsbelehrung beigefügt, so beträgt die Frist 1 Jahr ( 66 Absatz 2 SGG).

Es gilt der Amtsermittlungsgrundsatz: In der Regel wird ein externes Sachverständigengutachten eingeholt ( $\S 103$, 106 SGG). Will und/oder muss der Kläger ein solches entkräften, so besteht die Möglichkeit, die Einholung eines weiteren Gutachtens zu beantragen, dies unter Benennung eines bestimmten Arztes ( $\$ 109$ SGG). Diese Vorgehensweise stellt eine Durchbrechung des Amtsermittlungsgrundsatzes dar und erfolgt deshalb auch - im Gegensatz zu gerichtlicherseits eingeholten Gutachten - auf Kosten des Klägers (ausnahmsweise können die Kosten von der Staatskasse übernommen werden, der Antrag ist aber nur nachträglich möglich [6]).

Um die Kosten des Verfahrens, einschließlich des beauftragten Rechtsanwalts tragen zu können, empfiehlt sich unbedingt der Abschluss einer Rechtsschutzversicherung. Wirtschaftlich schwache Kläger können Prozesskostenhilfe beantragen.

Gegen ein Urteil eines Sozialgerichtes sind Berufung zum Landessozialgericht (LSG) und in Ausnahmefällen Revision zum BSG gegeben. Weitere Möglichkeiten der Verfahrensbeendigung sind das Anerkenntnis der Beklagten, der Vergleich und die Klagerücknahme. Hier tritt sofort Rechtskraft ein.

\section{Aktuelle Entwicklungen}

Mittlerweile liegt zum Thema Adipositas der neue Begutachtungsleitfaden des Medizinischen Dienstes des Spitzenverbandes Bund der Krankenkassen e.V. (MDS) vor. Dieser Leit- 
faden wurde am 25. November 2009 von der Konferenz der Leitenden Ärzte und Ärztinnen verabschiedet und am 21. Dezember 2009 vom Beirat für MDK-Koordinierungsfragen zur zukünftigen Anwendung empfohlen. Dort wird die chirurgische Therapie der Adipositas für Patienten mit einem Body Mass Index (BMI) von über $60 \mathrm{~kg} / \mathrm{m}^{2}$ (zu dieser Gruppe gehört auch die Klägerin) als alternativlos dargestellt und entsprechend empfohlen [7]. Zitat:

«Bei Vorliegen eines BMI $>60 \mathrm{~kg} / \mathrm{m}^{2}$ ist nach der aktuell verfügbaren Literatur eine relevante Gewichtsbeeinflussung auch unter multimodaler Therapie nicht zu erwarten. Es ist in dieser Konstellation nur sicherzustellen, dass keine Kontraindikation oder keine vorrangig zu behandelnden Grunderkrankungen vorliegen und dass eine ernährungsmedizinische Betreuung als Vorbereitung auf die postoperative Phase stattgefunden hat."

Des Weiteren liegt seit April 2010 die neue S3-Leitlinie «Chirurgie der Adipositas» vor. Diese Leitlinie führt - und das ist neu - ein weiteres Beurteilungskriterium ein, nämlich den Begriff der «geringen Erfolgsaussichten» [8]. Lassen demnach "Art und/oder Schwere der Krankheit bzw. psychosoziale Gegebenheiten bei Erwachsenen annehmen, dass eine chirurgische Therapie nicht aufgeschoben werden kann oder die konservative Therapie ohne Aussicht auf Erfolg ist, kann in Ausnahmefällen auch primär eine chirurgische Therapie durchgeführt werden.»

Schließlich: Die chirurgische Behandlung (Remission/Heilung?) des Diabetes mellitus Typ 2 ist in aller Munde. Auch bei den Gerichten hat sich mittlerweile herumgesprochen, dass extrem adipöse (und insbesondere insulinpflichtige) Diabetiker auf konservativem Wege nicht signifikant und nachhaltig an Gewicht verlieren können. Das Landgericht Wiesbaden hat in einem spektakulären Urteil die Metabolische Chirurgie als originäre Diabetesbehandlung für einen PKV-Patienten mit einem vertraglichen Leistungssausschluss für «Maßnahmen zur Gewichtsreduktion» anerkannt (Urteil vom 5. Mai 2010, Az. 5 O 263/08, noch nicht rechtskräftig).

\section{Exkurs § 13 SGB V}

Möglich ist es, hinsichtlich der Kosten einer adipositaschirurgischen Operation in Vorleistung zu treten und die Krankenkasse im Nachgang auf Erstattung in Anspruch zu nehmen, $\S 13$ Absatz 3 SGB V. Die Vorschrift lautet:

"Konnte die Krankenkasse eine unaufschiebbare Leistung nicht rechtzeitig erbringen (1. Alternative, Anmerkung des Autors) oder hat sie eine Leistung zu Unrecht abgelehnt (2. Alternative, Anmerkung des Autors) und sind dadurch Versicherten für die selbstbeschaffte Leistung Kosten entstanden, sind diese von der Krankenkasse in der entstandenen Höhe zu erstatten, soweit die Leistung notwendig war.»
Sind die Voraussetzungen dieser Vorschrift erfüllt, so wandelt sich der Anspruch auf Gewährung der Operation als Sachleistung (Sachleistungsanspruch) in einen Erstattungsanspruch, der zunächst außergerichtlich und dann gerichtlich geltend gemacht werden kann.

Zuvor allerdings muss sich der Versicherte in zumutbarem Umfang um die Gewährung der streitigen Behandlung als Sachleistung bemüht haben. Der Betroffene muss sich also gedulden, bis die Entscheidung über seinen Antrag auf Kostenübernahme schriftlich vorliegt. Die Entscheidung über einen erhobenen Widerspruch muss er dagegen nicht abwarten [9].

Die wichtigste Anspruchsvoraussetzung ist die im Gesetzestext nicht ausdrücklich genannte Kausalität zwischen der Ablehnung der Leistung durch die Krankenkasse und der Selbstbeschaffung dieser Leistung durch den Versicherten: Der Entschluss zur Durchführung der OP darf zeitlich nicht vor der Ablehnungsentscheidung der Krankenkasse liegen. Unbedingt vermieden werden muss die Festlegung eines OPTermins vor der Ablehnung bzw. die verfrühte Unterzeichnung einer Honorarvereinbarung [10]. Unbedenklich ist die im Vorfeld geführte Korrespondenz mit dem Operateur und/ oder dem Krankenhaus, soweit hier nur allgemeine Informationen eingeholt und ausgetauscht werden. Wie in diesem $\mathrm{Zu}$ sammenhang die Einholung eines Kostenvoranschlags durch den Patienten zu werten ist, ist noch ungeklärt [11].

Die Höhe des Anspruches entspricht den tatsächlich verauslagten Kosten. Eine wie auch immer geartete Kappung, etwa auf den sogenannte Kassensatz (gemäß $§ 7$ KH EntG DRG K04A) hat der Gesetzgeber durch die Formulierung «... in der entstandenen Höhe zu erstatten» explizit ausgeschlossen. Auch Zinsen, etwa für die Inanspruchnahme eines Bankkredites, sind Teil des Erstattungsanspruchs.

Vorteil dieser Vorgehensweise: Ist «nur» eine Geldsumme streitig, so kann zwischen den Parteien des Rechtsstreits ein Vergleich geschlossen werden.

\section{Exkurs § 52 SGB V}

Immer wieder versuchen Krankenkassen die Adipositas als selbstverschuldete Krankheit einzustufen. Auf diese Weise sollen Kosten, die aufgrund von Komplikationen nach einer privat bezahlten adipositaschirurgischen Operation entstanden sind, auf den Versicherten abgewälzt werden.

$\S 52$ SGB V bestimmt:

«Haben sich Versicherte eine Krankheit vorsätzlich (...) zugezogen, kann die Krankenkasse sie an den Kosten der Leistungen in angemessener Höhe beteiligen und das Krankengeld ganz oder teilweise für die Dauer dieser Krankheit versagen oder zurückfordern.»

«Haben sich Versicherte eine Krankheit durch eine medizinisch nicht indizierte ästhetische Operation, 
eine Tätowierung oder ein Piercing zugezogen, hat die Krankenkasse die Versicherten in angemessener Höhe zu beteiligen und das Krankengeld für die Dauer dieser Behandlung ganz oder teilweise zu versagen oder zurückzufordern.»

Diese Norm durchbricht den Grundsatz, wonach im Interesse der Versicherten und der Allgemeinheit Leistungen grundsätzlich ohne Rücksicht auf die Krankheitsursachen zu gewähren sind [12]. Sie konkretisiert erstmals das in $\S 1$ Satz 2 SGB V niedergelegte Leitbild der Eigenverantwortlichkeit der Versicherten.

Neben einem ursächlichen Kausalzusammenhang zwischen einem aktiven Tun (oder einem Unterlassen) und der behandlungsbedürftigen Krankheit verlangt $\S 52$, dass sich der Vorsatz (mindestens in Form einer billigenden Inkaufnahme) auch auf die Verursachung einer Krankheit bezieht. Einen solchen Vorsatz wird man in Fällen bloßer gesundheitsschädlicher Lebensführung (exzessives Rauchen, Alkoholabusus, übermäßige Nahrungsaufnahme) regelmäßig nicht annehmen können.
Schließlich entspräche eine solche Auslegung auch nicht der gesetzgeberischen Intention: Die Solidargemeinschaft soll für jene Maßnahmen nicht eintreten müssen, die rein kosmetischen Charakter haben und/oder lediglich der Erhöhung der Lebensqualität dienen [13].

Im Falle der Adipositaschirurgie heißt das: Hat ein Facharzt einen Patienten gemäß den Leitlinien der Fachgesellschaften ausgewählt, die medizinische Indikation gestellt und schließlich operiert, so ist im Falle auftretender Komplikationen für eine Anwendung des $§ 52$ SGB V kein Raum, dies unabhängig davon, ob eine Krankenkasse die medizinische Indikation bestreitet oder nicht. Denn: So lange die medizinische Indikation streitig ist, ist das Tatbestandsmerkmal «medizinisch nicht indiziert» nicht erfüllt.

\section{Disclosure Statement}

Keine. Herr Werner ist nur und ausschließlich dem Wohl seiner Mandanten verpflichtet.

\section{Literatur}

1 Rieber N, Hilbert A, Teufel M, Giel KE, Warschburger P, Zipfel S: Gewichtsstabilisierung nach Gewichtsreduktion. Eine kritische Bestandsaufnahme zu Prädiktion und Intervention. Adipositas 2010;4115-124.

- Sjöström L, Narbro K, Sjöström CD, Karason K, Larsson B, Wedel H, Lystig T, Sullivan M, Bouchard C, Carlsson B, Bengtsson C, Dahlgren S, Gummesson A, Jacobson P, Karlsson J, Lindroos AK, Lönroth H, Näslund I, Olbers T, Stenlöf K, Torgerson J, Agren G, Carlsson LM; Swedish Obese Subjects Study: Effects of bariatric surgery on mortality in Swedish obese subjects. N Engl J Med 2007;357:741-752.
3 Buchwald H, Oien DM: Metabolic/bariatric surgery worldwide 2008. Obes Surg 2009;19:1605-1611.

4 Kasseler Kommentar zum Sozialversicherungsrecht, § 137c, Rn. 3, 4.

5 Bundessozialgericht, Urteil vom 19. Februar 2003, Az. B 1 KR 1/02 R, www.bundessozialgericht.de.

6 Exemplarisch: Landessozialgericht NordrheinWestfalen, Beschluss vom 18. Dezember 2007, Az. L 2 B 17/07 KN.

7 Medizinischer Dienst des Spitzenverbandes Bund der Krankenkassen: Begutachtungsleitfaden «Bariatrische Chirurgie (Adipositaschirurgie) bei Erwachsenen. 21. Dezember 2009. www.mds-ev.org (zur Zeit nicht abrufbar).
8 S3-Leitlinie «Chirurgie der Adipositas» der Chirurgischen Arbeitsgemeinschaft für Adipositastherapie der Deutschen Gesellschaft für Allgemein- und Vizeralchirurgie in Zusammenarbeit mit der Deutschen Adipositasgesellschaft, 2009. www.adipositas-gesellschaft.de/leitlinien.php.

9 Krauskopf, Soziale Krankenversicherung, Kommentar zum SGB V, § 13, Rn. 31 m.w.N.

10 Zuletzt bestätigt durch das LSG Baden-Württemberg, Urteil vom 23. Februar 2010, Az. L 11 KR 4761/09).

11 Zur Zeit streitig vor dem SG Fulda, Az. S 11 KR $157 / 09$.

12 So schon BSGE 18, S. 257f. und BSGE 59, S. 119.

13 Siehe z. B. Kasseler Kommentar zum Sozialversicherungsrecht, Verlag C. H. Beck, § 52 SGB V, Rn. 23f. 